\title{
Lateral column lengthening augmented by medial split tibialis posterior tendon advancement for the treatment of symptomatic flexible flatfeet
}

\author{
Amin Abdelrazek Youssef Ahmed*, Mina Michael
}

Department of Orthopedic Surgery, Faculty of Medicine, Alexandria University, Alexandria, Egypt

Received: 22 February 2019

Revised: 16 May 2019

Accepted: 17 May 2019

\section{*Correspondence:}

Dr. Amin Abdelrazek Youssef Ahmed,

E-mail: aminrazek@yahoo.com

Copyright: () the author(s), publisher and licensee Medip Academy. This is an open-access article distributed under the terms of the Creative Commons Attribution Non-Commercial License, which permits unrestricted non-commercial use, distribution, and reproduction in any medium, provided the original work is properly cited.

\begin{abstract}
Background: Management of Idiopathic flexible pes planus (IFPP) is debatable. Surgery is rarely indicated for flexible flatfoot. The goal of the surgery should be always to treat symptomatic patients and not to alter the shape of the foot. There are numerous options for surgical treatment in children and adolescents including subtalar arthroereisis or osteotomy with or without soft tissue procedures.

Methods: Between June 2013 to December 2017, twenty eight feet in sixteen patients (9 boys, 7 girls) were included in this study. Twelve cases were operated bilaterally, three in the right foot and one in the left side. The mean age of the patients was $8.36 \pm 1.704$ years (6-12 years). Calcaneal lengthening osteotomy was done with an oscillating saw about $1.5 \mathrm{~cm}$ proximal to the calcaneocuboid joint. Usually, $8-10 \mathrm{~mm}$ autologous bone graft from iliac crest was sufficient and fixed by $\mathrm{K}$ wires. Half of the tibialis posterior tendon after splitting with a periosteal flap from the navicular was then advanced distally until clinical restoration of the medial arch was then performed.

Results: Radiographic analysis revealed significant improvements in talo-first metatarsal, calcaneal pitch, talocalcaneal angles in lateral radiographs, and talo-first metatarsal and talonavicular coverage angles in AP radiographs. All patients were evaluated at final follow-up visits. Preoperative mean AOFAS score increased significantly from $64.04 \pm 8.867$ (range: 50 to 75 ) to $94.11 \pm 3.765$ (90-100).

Conclusions: It is concluded that Evans calcaneal lengthening osteotomy augmented by spilt tibialis posterior tendon advancement is an excellent procedure in the management of IFPP.
\end{abstract}

Keywords: Flat foot, Evans osteotomy, Calcaneal lengthening, Tibialis posterior, Advancement

\section{INTRODUCTION}

Idiopathic flexible pes planus (IFPP) is a common problem affecting children and adolescents. ${ }^{1}$ This condition is unfortunately remains poorly understood mainly due to the large volume of conflict to the extent that some health care providers consider flatfoot as a variation of the normal of the foot. ${ }^{2}$ There is no consensus on an accepted definition of a normal medial longitudinal arch but generally, a flatfoot is defined as a foot with a decrease in or absent longitudinal arch while weightbearing. ${ }^{2}$
Management of this problem is debatable. It begins with conservative measures as assurance and education of the patient and parents, Foot orthoses, stretching, shoe and activity modifications, casting, weight reduction in obese patients, and non steroidal drugs. ${ }^{2,3}$ Surgery is rarely indicated for flexible flatfoot. The goal of the surgery should be always to treat symptomatic patients and not to alter the shape of the foot which does not necessarily create problems because the risks associated with surgery must be considered against an innocent natural history of the foot shape itself. ${ }^{2}$ 
There is still no strict information about indications for surgical treatment but in general, patients who did not respond to conservative treatment may be candidates for surgical treatment. There are numerous options for surgical treatment in children and adolescents including subtalar arthroereisis or osteotomy with or without soft tissue procedures. ${ }^{4-7}$

The objective of this prospective study was to evaluate the results of management of flexible flat foot by lateral column calcaneal lengthening osteotomy augmented by medial split tibialis posterior tendon advancement.

\section{METHODS}

This prospective study was approved by the local ethical committee of the university and has therefore been performed in accordance with the pertinent ethical guidelines (i.e. Declaration of Helsinki, as laid down in 1964 and revised in 2008). The study was performed on children and adolescents with symptomatic flatfeet after taking patients and parents' consent.

Between June 2013 to December 2017, twenty eight feet in sixteen patients ( 9 boys, 7 girls) were included in this study. Twelve cases were operated bilaterally, three in the right foot and one in the left side. Patients were treated at
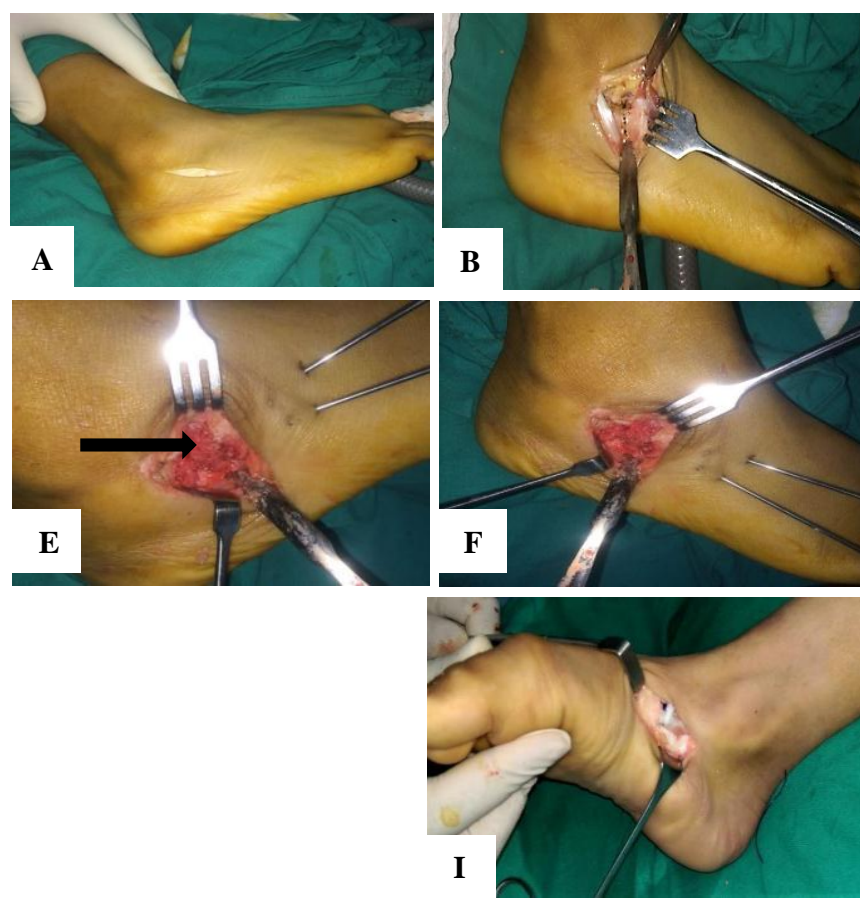

Elhadra University Hospital where all patients had been treated conservatively for at least one year. Patients with spastic flat foot were excluded from the study.

Pre operative clinical and radiological assessments were done in all patients including standing anteroposterior (AP) and lateral radiographs of the foot and ankle. Multiple angles were measured pre and post operative to assess radiological improvement after surgery.

On AP view, The following axes were drawn on the weight-bearing anteroposterior view: axis of the talus, axis of the calcaneus and longitudinal axis of the first metatarsal after that, the following angles were measured on this view: talocalcaneal divergence (normal $20^{\circ}-25^{\circ}$ ), talo first metatarsal angle (T 1 st MT) (normal $0^{\circ}-5^{\circ}$ ). The talonavicular coverage angle, taken as a percentage, was made by the lines perpendicular to the lines passing through the medial and lateral edges of the talar and navicular joint surfaces.

The following axes were drawn on the lateral view: longitudinal axis of the talus, axis of the calcaneus, and longitudinal axes of first metatarsal then the following angles were measured: calcaneal pitch (normal $15^{\circ}-20^{\circ}$ ), T 1st MT angle or Méary's angle (normal $0^{\circ}-10^{\circ}$ ) and talocalcaneal angle (normal $35^{\circ}-40^{\circ}$ ).
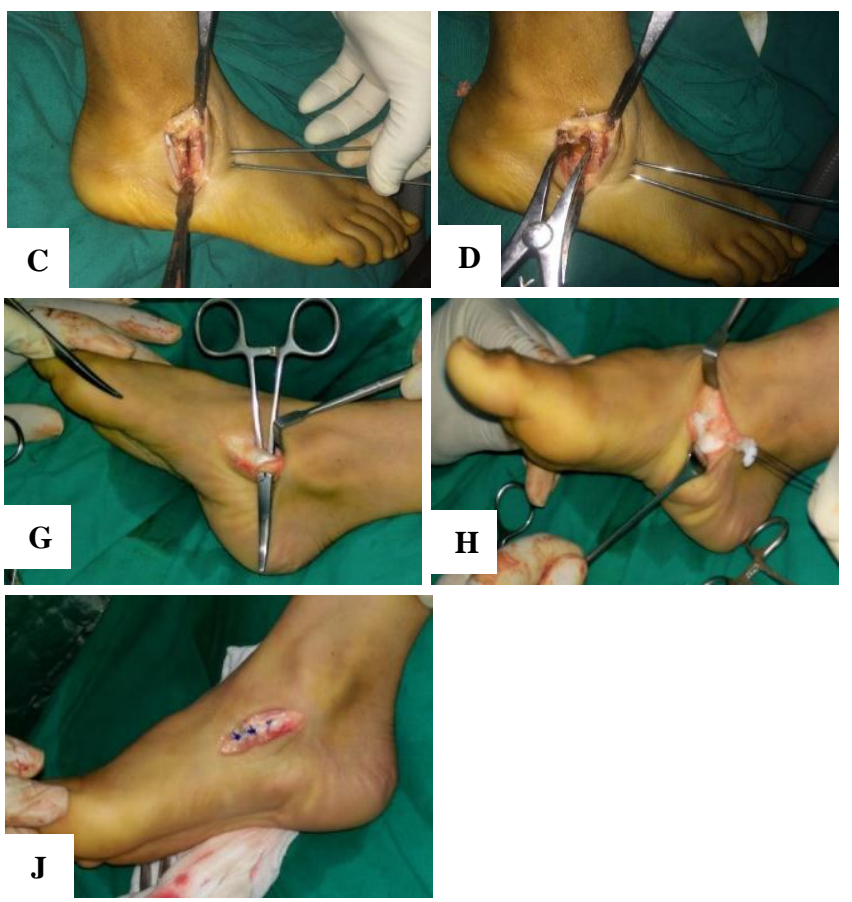

Figure 1: Operative procedure used in management RT flat foot. (A) Skin incision. (B) The site of the osteotomy, about $1.5 \mathrm{~cm}$ proximal to the calcaneocuboid joint passing between the anterior and middle facets of the calcaneus.

(C) Calcaneal osteotomy. (D) Using lamina spreader to determine the adequate graft size needed. (E) Putting autogenous tricortical iliac bone graft at the osteotomy site. (F) Fixation of the osteotomy by advancing the

previously inserted wires into the graft and the proximal calcaneal fragment. (G) Medial approach along the posterior tibial tendon to its insertion into the navicular bone. $(\mathrm{H})$ Splitting of the tibialis posterior tendon into two halves. (I) Advancement of half of the splitted tendon distally into the navicular. (J) Closure of the tendon sheath. 


\section{Operative procedure, (Figure 1)}

All patients were operated under general anesthesia in supine position with inflation of thigh tourniquet on the side of the operation. Calcaneal lengthening osteotomy was done with an oscillating saw about $1.5 \mathrm{~cm}$ proximal to the calcaneocuboid joint passing between the anterior and middle facets of the calcaneus. Calcaneocuboid joint was temporarily fixed with one or two K-wires passing from the dorsum of the foot into the cuboid traversing the calcaneocuboid joint into the distal calcaneal fragment to guard against calcaneocuboid subluxation while opening the osteotomy site. Lamina spreader was used to determine the adequate graft size needed. The amount of distraction was determined by monitoring the restoration of medial arch and neutral heel. Usually, 8-10 mm autologous bone graft from iliac crest was sufficient and fixed by advancing the previously inserted wires into the graft and the proximal calcaneal fragment.

Medial approach along the posterior tibial tendon to its insertion into the navicular bone was done. Half of the tendon after splitting with a periosteal flap from the navicular was then advanced distally until clinical restoration of the medial arch was then performed.

Closure of medial and lateral incisions after good haemostasis was performed. Below knee cast was then applied for at least 6 weeks. The mean time of follow up was $22.57 \pm 7.876$ months range from 12 to 36 months.
The AOFAS hindfoot and ankle scores were calculated for all patients preoperatively and at final follow-up examination.

\section{Statistical analysis}

Data were analyzed by using $\operatorname{SPSS}^{\mathrm{R}}$ software (Statistical package for social science for personal computers, IBM, Armonk, New York) using Pearson Chi-square $\mathrm{X}^{2}$ test and comparing means, Qualitative data were described using numbers and percentage. Quantitative data were expressed as mean $\pm \mathrm{SD}$ and $\mathrm{p}<0.05$ considered significant.

\section{RESULTS}

The mean age of the patients were $8.36 \pm 1.704$ years (612 years). Preoperative mean AOFAS score increased significantly from $64.04 \pm 8.867$ (range: 50 to 75 ) to 94.11 \pm 3.765 (range: 90 to100). All patients and their parents were satisfied with surgery results and twelve patients with symptomatic bilateral deformities asked for surgery to correct the other foot. A statistical description of the case series is depicted in (Table 1).

Radiographic analysis revealed significant improvements in talo-first metatarsal, calcaneal pitch, talocalcaneal angles in lateral radiographs, and talo-first metatarsal and talonavicular coverage angles in AP radiographs, (Table 2).

Table 1: Statistical description of the case series.

\begin{tabular}{|lll|}
\hline Variable & & \multicolumn{1}{l|}{$\begin{array}{c}\text { Mean (range) } \\
\text { Or count (\%) }\end{array}$} \\
\hline Age (years) & & $8.36 \pm 1.704(6-12)$ \\
\hline \multirow{2}{*}{ Sex } & Male & $9(56.25)$ \\
\hline \multirow{2}{*}{ Side } & Female & $7(43.75)$ \\
\hline Preoperative AOFAS & Bilateral & $12(75)$ \\
\hline Postoperative AOFAS & Right & $3(18.75)$ \\
\hline Follow up period (months) & & $1(6.25)$ \\
\hline
\end{tabular}

Table 2: Radiographic analysis of patients.

\begin{tabular}{|c|c|c|c|}
\hline & & $\begin{array}{l}\text { Preoperative value (degrees) } \\
\text { Mean } \pm \text { SD (range) }\end{array}$ & $\begin{array}{l}\text { Postoperative value (degrees) } \\
\text { Mean } \pm \text { SD (range) }\end{array}$ \\
\hline \multirow{2}{*}{ AP view } & TN coverage angle & $\begin{array}{l}26.50 \pm 5.594 \\
(20-35)\end{array}$ & $\begin{array}{l}7.71 \pm 4.529 \\
(3-16)\end{array}$ \\
\hline & T 1st MT & $\begin{array}{l}16.71 \pm 4.293 \\
(12-26)\end{array}$ & $\begin{array}{l}7.00 \pm 2.880 \\
(4-12)\end{array}$ \\
\hline \multirow{3}{*}{$\begin{array}{l}\text { Lateral } \\
\text { view }\end{array}$} & Cal pitch & $\begin{array}{l}10.25 \pm 1.602 \\
(7-12)\end{array}$ & $\begin{array}{l}18.93 \pm 2.638 \\
(14-22)\end{array}$ \\
\hline & TC angle & $\begin{array}{l}46.00 \pm 2.956 \\
(42-50)\end{array}$ & $\begin{array}{l}39.71 \pm 3.980 \\
(35-46)\end{array}$ \\
\hline & $\mathrm{T}$ 1st MT & $\begin{array}{l}16.29 \pm 3.680 \\
(12-22)\end{array}$ & $\begin{array}{l}6.50 \pm 3.437 \\
(3-12)\end{array}$ \\
\hline
\end{tabular}



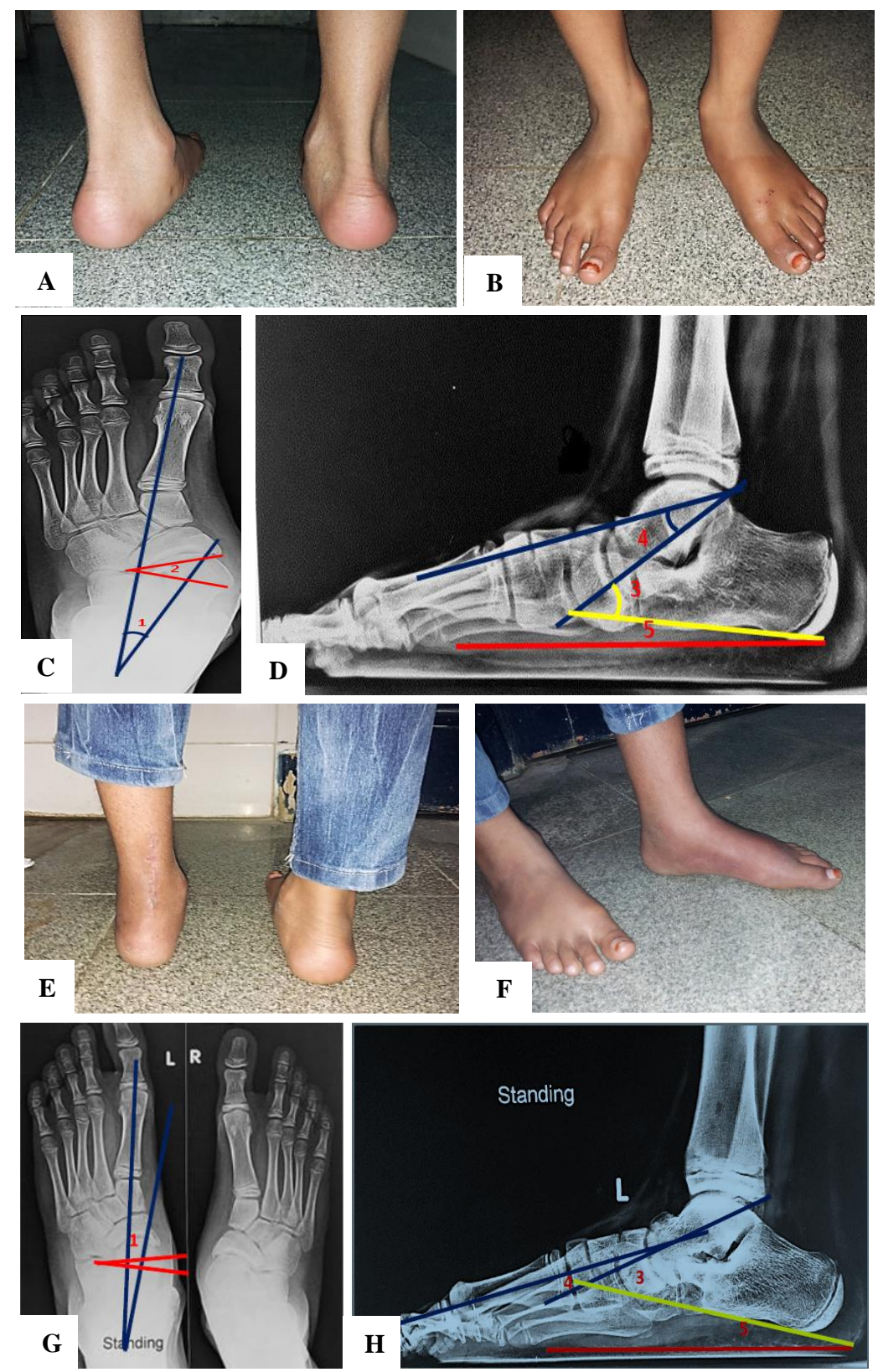

Figure 2: Clinical and radiological presentation of an 11 year old girl with bilateral flat feet. (A, B) Preoperative photograph of the patient standing. (C) Preoperative X ray of the same patient, AP standing view of the left foot showing: 1 ( $\left.1^{\text {st }} \mathrm{MT}=14\right)$ and 2 (TN coverage angle=35). (D) Preoperative $X$ ray of the same patient, lateral standing view of the left foot showing: 3 (TC angle=42), 4 (T 1st MT=22) and 5 (Cal pitch angle =7).

(E, F) Photograph of the patient after correction of the left foot. (G) Postoperative $X$ ray of the same patient after correction of the left foot, AP standing view showing: 1 ( $T$ 1st MT=10) and 2 (TN coverage angle=12).

(H) Postoperative X ray of the same patient, lateral standing view showing: 3 (TC angle=35), 4 (T 1st MT=8) and 5 (Cal pitch angle=14).

\section{DISCUSSION}

There is still no optimal standard algorithm for treatment of symptomatic flexible flatfoot in children and adolescents. The only accepted indication for surgical treatment is failure of conservative treatments. ${ }^{8}$ However, the decision to operate also depends on the surgeon knowledge and experience. We believe that symptomatic patients are candidates for surgical correction to relieve symptoms and avoid permanent deformities. ${ }^{9}$

There are many surgical procedures for treatment of this deformity including subtalar arthroereisis or bony osteotomies with or without soft tissue procedures. Mosca advocated the Evans calcaneal osteotomy when surgery is indicated and supported his recommendation 
with favorable clinical observations. ${ }^{3}$ Verheyden et al in 1997 evaluated subtalar arthroereisis with the Teflon sinus tarsi spacer and they reported a high incidence of postoperative foot pain and a high rate of implant dislocation. $^{10}$ Osteotomies include lateral calcaneal lengthening osteotomy described by Evans, Cotton's medial cuneiform osteotomy and medializing calcaneal osteotomy. We preferred to do combined Evans calcaneal lengthening osteotomy with soft tissue procedures.

After calcaneal osteotomy, we found significant improvement in calcaneal pitch, talonavicular coverage, and in talo first metatarsal and talocalcaneal angles. Improvements achieved in the present study were not limited to radiography results. There were also significant improvements in AOFAS scores. In addition, all patients and their families were satisfied with surgery results and 12 patients were operated on bilaterally. These results coincides with results of other authors. ${ }^{9,11,12}$

Evans calcaneal osteotomy is a powerful procedure that provides triplanar correction. Correction occurs in the transverse as well as the sagittal and frontal planes. It lengthens the lateral column and reduces the forefoot abduction and transverse plane deformity. In addition, realignment of the midtarsal joint is achieved and calcaneal eversion and frontal plane deformity is reduced. This procedure also places tension on the long plantar ligaments and provides significant arch elevation and stabilization reducing the sagittal plane deformity. In 1995, Mosca reported correction of all components with the advantage of restoration of the function of the subtalar joint, and theoretically, the ankle and mid-tarsal joints were protected from early degenerative arthrosis by avoiding arthrodesis. ${ }^{13}$

The graft size and source whether auto or allograft is a point for discussion. The main concern of calcaneal lengthening is development of degenerative changes at the calcaneocuboid joint. Cooper et al showed that elongating the lateral column of normal feet raised the pressure across the calcaneocuboid joint. ${ }^{14}$ Momberger et al determine the change in pressure through the calcaneocuboid joint after an Evan's-type calcaneal lengthening osteotomy and stated that there was no significant change in pressure between the flatfoot and the corrected foot, even they found that the peak pressures were actually lower in the corrected foot than in the flatfoot in some cases. ${ }^{15}$ These findings indicate that Evan's calcaneal lengthening osteotomy does not increase pressure across the calcaneocuboid joint further than physiologic loads in the flatfoot.

Furthermore, CC joint sublaxation has been reported to be a probable complication after calcaneal lengthening. ${ }^{13,15,16} \mathrm{Ji}$-Yong Ahn et al described that CC joint subluxation happened in all cases after a calcaneal lengthening procedure regardless of pinning across the $\mathrm{CC}$ joint with a $\mathrm{K}$-wire, however they believe that the percentage of $\mathrm{CC}$ joint subluxation could improve with time without the occurrence of osteoarthritis. ${ }^{17}$

Many reports had driven surgeons to try to avoid CC joint subluxation by decreasing the bone graft size. ${ }^{14,18,19} \mathrm{Xia}$ et al proposed that the ideal bone graft size is $8 \mathrm{~mm}$ for calcaneal lengthening. ${ }^{20}$ In our study, we used bone graft ranged from 8 to $10 \mathrm{~mm}$ depending on the degree of deformity with fixation of cc joint by $\mathrm{K}$. wires to guard against subluxation.

The bone graft may be allogenic tricortical iliac crest graft or autogenous tricortical graft. We use autogenous graft due to unavailability of allogenic graft and also for better incorporation of autogenous graft. No patient developed non union in our cases. Necip et al reported one case of non united graft. ${ }^{9}$ They used allogenic graft in this case and they reported that non-union might be because of the longer time required for allograft to become incorporated.

Additional procedures are usually required to stabilize the medial column either cotton medial cuneiform Osteotomy or soft tissue procedures. Cotton osteotomy reduces pressure in lateral column of the foot. ${ }^{21}$ Reduction in pressure is thought to result in the alleviation of lateral column pain. Necip et al did cotton osteotomy in only 5 of 21 feet and they did not document lateral column pain, even in those who did not carry out cotton osteotomy. ${ }^{9}$ We used split tibialis posterior tendon advancement to stabilize the medial arch after Evans osteotomy. In few cases, the tibialis posterior tendon becomes degenerated and flexor digitorum longus transfer can be used as in adults but in our cases we didn't find any case of tibialis posterior degeneration may be due to the young age of our patients.

\section{CONCLUSION}

It is concluded that Evans calcaneal lengthening osteotomy augmented by spilt tibialis posterior tendon advancement is an excellent procedure in the management of symptomatic idiopathic flexible pes planus (IFPP).

\section{Funding: No funding sources \\ Conflict of interest: None declared}

Ethical approval: The study was approved by the institutional ethics committee of Alexandria Main University Hospital

\section{REFERENCES}

1. Blitz NM, Stabile RJ, Giorgini RJ, DiDomenico LA. Flexible pediatric and adolescent pes planovalgus: conservative and surgical treatment options. Clin Podiatr Med Surg, 2010;27:59-77.

2. Mosca VS. Flexible flatfoot in children and adolescents. J Child Orthop. 2010;4:107-21. 
3. Yeagerman SE, Cross MB, Positano R, Doyle SM. Evaluation and treatment of symptomatic flatfoot. Curr Opin Pediatr. 2011;2:60-7.

4. Roth S, Sestan B, Tudor A, Ostojic Z, Sasso A, Durbesic A. Minimally invasive calcaneo-stop method for idiopathic, flexible pes planovalgus in children. Foot Ankle Int. 2007;28:991-5.

5. Jay RM, Din N. Correcting pediatric flatfoot with subtalar arthroereisis and gastrocnemius recession:a retrospective study. Foot Ankle Spec. 2013;6:101-7.

6. Oh I, Williams BR, Ellis SJ, Kwon DJ, Deland JT. Reconstruction of the symptomatic idiopathic flatfoot in adolescents and young adults. Foot Ankle Int, 2011;32:225-32.

7. Akimau P, Flowers M. Medium term outcomes of planovalgus foot correction in children using a lateral column lengthening approach with additional procedures 'a la carte'. Foot Ankle Surg, 2014;20:26-9.

8. Moraleda L, Salcedo M, Bastrom TP, Wenger DR, Albinana J, Mubarak SJ. Comparison of the calcaneo-cuboid-cuneiform osteotomies and the calcaneal lengthening osteotomy in the surgical treatment of symptomatic flexible flatfoot. J Pediatr Orthop. 2012;32:821-9.

9. Necip S, Tahir O, Mehmet FG, Huseyin B, Gokhan $\mathrm{K}$, Ata Can. Surgical treatment results for flexible flatfoot in adolescents. Acta Orthopaedica et Traumatologica Turcica 2016;50:655-9.

10. Verheyden F, Vanlommel E, Van Der Bauwhede J, Fabry G, Molenaers G. The sinus tarsi spacer in the operative treatment of flexible flat feet. Acta. Orthop. Belg. 1997;63:305-9.

11. Oh I, Williams BR, Ellis SJ, Kwon DJ, Deland JT. Reconstruction of the symptomatic idiopathic flat foot in adolescents and young adults. Foot Ankle Int. 2011;32:225-32.

12. Akimau P, Flowers M. Medium term outcomes of planovalgus foot correction in children using a lateral column lengthening approach with additional procedures 'a la carte'. Foot Ankle Surg. 2014;20:26-9.

13. Mosca VS. Calcaneal lengthening for valgus deformity of the hindfoot. Results in children who had severe, symptomatic flatfoot and skew foot. J Bone Joint Surg Am 1995;77:500-12.

14. Cooper P, Nowak MD, Shaer J. Calcaneocuboid joint pressures with lateral column lengthening (Evans) Procedure. Foot and Ankle Int. 1997;18(4):199-205.

15. Momberger N, Morgan JM, Bachus KN, West JR. Calcaneocuboid joint pressure after lateral column lengthening in a cadaveric planovalgus deformity model. Foot Ankle Int. 2000;21(9):730-5.

16. Adams SB Jr, Simpson AW, Pugh LI, Stasikelis PJ. Calcaneocuboid joint subluxation after calcaneal lengthening for planovalgus foot deformity in children with cerebral palsy. J Pediatr Orthop. 2009;29(2):170-4.

17. Ahn JY, Lee HS, Kim CH, Yang JP, Park SS. Calcaneocuboid Joint Subluxation After the Calcaneal Lengthening Procedure in Children. Foot Ankle Int, 2014;35(7):677-82.

18. Hintermann B, Valderrabano V, Kundert HP. Lengthening of the lateral column and reconstruction of the medial soft tissue for treatment of acquired flatfoot deformity associated with insufficiency of the posterior tibial tendon. Foot Ankle Int. 1999;20(10):622-9.

19. Sands A, Early J, Harrington RM, Tencer AF, Ching RP, Sangeorzan BJ. Effect of variations in calcaneocuboid fusion technique on kinematics of the normal hindfoot. Foot Ankle Int. 1998;19(1):1925.

20. Xia J, Zhang P, Yang YF, Zhou JQ, Li QM, Yu GR. Biomechanical analysis of the calcaneocuboid joint pressure after sequential lengthening of the lateral column. Foot Ankle Int. 2013;34(2):261-6.

21. Yarmel D, Mote G, Treaster A. The Cotton osteotomy:a technical guide. J Foot Ankle Surg. 2009;48:506-12.

Cite this article as: Ahmed AAY, Michael M. Lateral column lengthening augmented by medial split tibialis posterior tendon advancement for the treatment of symptomatic flexible flatfeet. Int J Res Orthop 2019;5:540-5. 